\title{
Research on Control Strategy for power equipment based on Operation Risk of Power System
}

\author{
Li Guanglei, a , Jing Xiangyang ${ }^{2, b *}$, Ma Mengchao ${ }^{3, c}, Q u ~ Y u y a 0^{4, d}$ and Yan \\ Yong ${ }^{5, e}$ \\ ${ }^{1}$ State Grid Shandong Electric Power Research Institute, Jinan, China \\ ${ }^{2}$ State Grid Shandong Electric Power Research Institute, Jinan, China \\ ${ }^{3}$ State Grid of China Technology College, Jinan, China \\ ${ }^{4}$ State Grid Dongying Power Supply Company, Dongying, China \\ ${ }^{5}$ State Grid Shouguang Power Supply Company, Shouguang, China \\ a lovexjtulgl@126.com, ${ }^{b} 214672717 @ q q . c o m,{ }^{c}$ qwi917a@sina.com, ${ }^{\mathrm{d}}$ swec@sohu.com, \\ Ig5@163.com
}

Keywords: condition-based maintenance; generation scheduling; maintenance plan.

Abstract. A maintenance schedule evaluation method for transmission and transformation equipment considering operation risk of power system is presented. A dimensionality reduction method is used to deal with large amount of constraints. The maintenance schedules are quantitatively evaluated in the aspect of economic, security and environment, and they are sorted with the index of maximum integrated benefits to provide quantitative evidence of decision-making for the maintenance managers. The Sequential Monte Carlo simulation is used to solve probability problems. The power system of Zibo is used as an example to verify the validity of the model and arithmetic presented in the paper.

\section{Introduction}

Condition-based maintenance relies on equipment performance and the suitable opportunity [1-4]. Compared with planned maintenance, condition-based maintenance can effectively reduce equipment maintenance costs, prolong service life of equipment and improve equipment reliability. In power grid, technologies of condition-based maintenance have been obtaining more and more attention and still changing [5-8].Traditional researches mostly focus on equipment condition monitoring and evaluation and making maintenance plans according to equipment status. However, with the expansion of grid size, the failure of any device not only affects itself, but also may make a greater negative impact on power system.

Therefore, arrangements for maintenance scheduling not only care about the state of the device itself, but also consider the impact of the whole system caused by equipment outage. Based on the above recognitions, optimization schemes of condition-based maintenance centered on reliabilities are quantitatively evaluated in the aspects of economic, security and environment. From the overall perspective of power grid operation, these schemes are used to provide quantitative evidence of decision-making for the maintenance managers.

\section{Overall design}

Sequential Monte Carlo simulation In view of time correlation of maintenance programs, Sequential Monte Carlo Simulation is used as the tool for this basis. Set the initial environment of power system, such as breakdown rate and repair rate of transmission components, initial state (running or outage) of transmission component, topology of the electric network, nodal generation or nodal load, transmission capacity constraints of transmission components. If results cannot converge or satisfy constraints, system needs to simulate the process of re-scheduling of power system by optimizing power flow, combine with various scheduling policies and obtain more realistic results. Finally respectively calculate reliability data of each node and the entire system. 
Optimization model in the random viable state, reducing dimension processing and solving under constraint conditions this paper based on DC Load Flow studies one system status and constructs the following optimization model:

$$
\begin{aligned}
& \min \sum_{i=1}^{N} P_{C, i} \\
& T_{l}=A\left(P_{G}+P_{C}-P_{D}\right)
\end{aligned}
$$

(2)

$$
\begin{aligned}
& \sum_{i \in N G} P_{G, i}+\sum_{i \in N C} P_{C, i}=\sum_{i \in N C} P_{D, i} \\
& P_{G}^{\min } \leq P_{G} \leq P_{G}^{\max }
\end{aligned}
$$

(4)

$$
\begin{aligned}
& 0 \leq P_{C} \leq P_{D} \\
& \left|T_{l}\right| \leq T_{l}^{\max }
\end{aligned}
$$

In the formulas listed above, $T_{l}$ represents the active power flowing through lines $\mathrm{l} ; T_{l}^{\max }$ represents the maximum active power through lines l; A represents incident matrix between branch and node; $P_{G}$ represents active power of generator; $P_{C}$ represents active power of virtual generator; $P_{D}$ represents node active load; $P_{G}^{\max }$ represents the maximum output of generator; $P_{G}^{\min }$ represents the minimum output of generator; $N G$ represents the number of generators; $N C$ represents the number of virtual generators.

The specific processes are shown as Fig.1. Fig. 1 shows that it's necessary to repeatedly modify and solve the minimum load-shedding model. The optimization problem adopts method of linear programming. To further improve the computational efficiency.

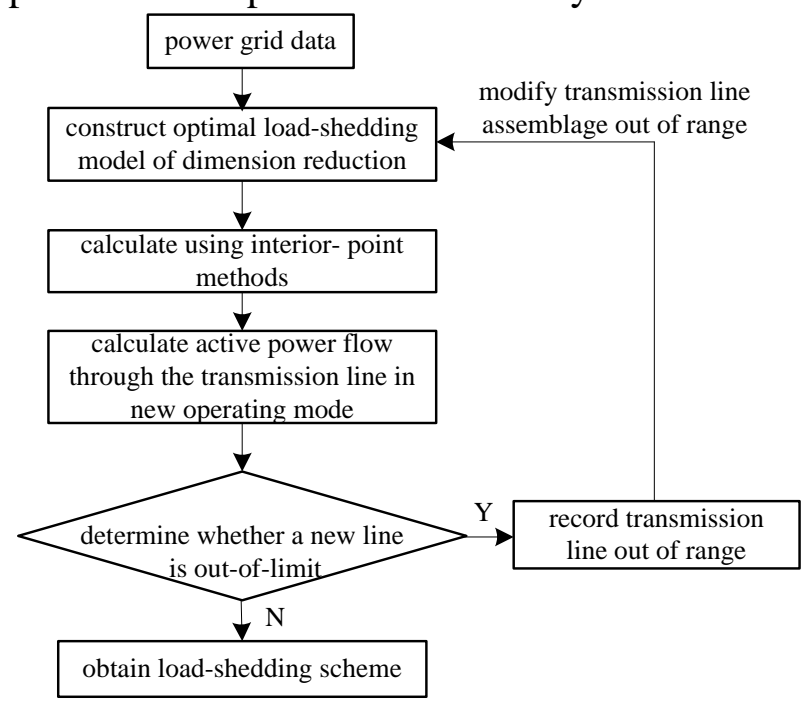

Fig.1 process of reducing dimension processing

Definitions of economic, security and environment indicators Definitions of economic, security and environment indicators are concretely expressed.The impact on security caused by fault must consider both personal and grid, respectively accounted for $50 \%$ of the weight. Impact factors of a variety of load loss on security are shown in following table 1 , reference to the recommended range in above guidelines. 
Table1 safety indicators

\begin{tabular}{|l|c|c|c|c|}
\hline \multirow{2}{*}{ load type } & \multicolumn{4}{|c|}{ safety factors } \\
\cline { 2 - 5 } & \multicolumn{2}{|c|}{ personal } & \multicolumn{2}{c|}{ grid } \\
\cline { 2 - 5 } & loss & $C_{k W h i 1}$ & loss & $C_{k W h i 2}$ \\
\hline first-class load & General & 7 & General & 4 \\
\hline second-class load & Major & 9 & Major & 7 \\
\hline third-class load & Large & 10 & Large & 10 \\
\hline
\end{tabular}

For each substation, according to the proportion of various types of load, $C_{k W h i}$ is calculated by the following formula (7).

$$
C_{k W h i}=\sum_{i=1}^{3} 0.5 \cdot\left(C_{k W h i 1}+C_{k W h i 2}\right) \cdot W_{i}
$$

In the formula (7), $i$ represents load type; 1 - first-class load, 2- second-class load, 3third-class load; $W_{i}$ represents the proportion of various types of load of substation $i$.

The probability of damage to the equipment can be obtained from simulation results; $E_{C 2 i}$ firstly needs to get the equipment damage degree of environmental impact (slight pollution, moderate pollution, and heavy pollution) according to equipment types, voltage level, working principle and other factors, then refers the following recommended range in "State Grid Corporation of power transmission equipment risk assessment guidelines".

Maintenance schedule evaluation and ordering Maintenance schedule evaluation needs to integrate its economic, environmental and security implications. Therefore, weighted sums of economic, environmental and safety losses are used in this article to evaluate the integrated consequences, calculated based on the following formula (8).

$$
R=W_{R 1} R_{1}+W_{R 2} R_{2}+W_{R 3} R_{3}
$$

In the formula (8), $R$ represents comprehensive risk of a maintenance schedule; $W_{R 1} 、 W_{R 2}$ and $W_{R 3}$ represent weight coefficient of economic, security and environmental factors, respectively valued for the 0.4,0.2,0.4 according to "State Grid Corporation of transmission and distribution equipment risk assessment guidelines". $R_{1}, R_{2}$ and $R_{3}$ respectively mean economic risk, security risk and environmental risk.

For the loss of all elements, the research not only consider equipment failure for the loss of their own, also take into account of effect of equipment failure on the whole grid. Because of different dimension of element loss in calculation, it's difficult to directly use the above weighted method. So, in the actual calculation, $R$ is replaced by sorting number of economic, environmental and security under this scene. The process is as follows: firstly separately calculate economic indicators, environment indicators and safety indicators under the scene then sort these values by the order from little to large, finally get the final composite indicator using its weighted serial number

\section{Analyses example}

Reliability evaluation Table 2 shows the results of reliability evaluation. Power Supply Reliability of Zibo Area Power System is $99.989 \%$, which is higher than the power supply reliability above 99.90\% in urban areas required by State Grid Corporation.

Table 2 reliability evaluation of Zibo Area Power System

\begin{tabular}{|c|c|}
\hline Evaluation Indexes & Results of reliability evaluation \\
\hline EENS (MW) & 1935 \\
\hline Economic Indicators (million) & 1674 \\
\hline Safety indicators (1) & 12481 \\
\hline Environmental indicators (1) & 950 \\
\hline
\end{tabular}

Maintenance schedule evaluation three kinds of maintenance schemes are calculated and compared. Maintenance scheme 1 is an actual maintenance scheme of a power supply company involving $110 \mathrm{kV}$ and above transmission and transformation primary equipment. This scheme 
includes projects such as maintenance, technical innovation and municipal transformation, involves 74 maintenance devices and totally invests 41.18 million. Maintenance scheme 2 is part of the equipment maintenance program, involves 45 maintenance devices and the total cost is 7.85 million; Maintenance scheme 3 is also part of the equipment maintenance program, involves 14 maintenance devices and the total cost is 36.77 million.

Table 3 maintenance schedule evaluation

\begin{tabular}{|c|c|c|c|}
\hline Type & Maintenance scheme 1 & Maintenance scheme 2 & Maintenance scheme 3 \\
\hline EENS(MW) & 1760 & 1894 & 2268 \\
\hline maintenance costs(million) & 41.18 & 7.85 & 36.77 \\
\hline economic indicators(million) & 56.33 & 23.81 & 53.34 \\
\hline safety indicators(1) & 11356 & 12221 & 14631 \\
\hline environmental indicators(1) & 835 & 887 & 319 \\
\hline comprehensive sequencing & 2 & 1 & 3 \\
\hline
\end{tabular}

The best order is maintenance scheme 1, while maintenance scheme 3 is the worst. These show that the actual $110 \mathrm{kV}$ and above transmission and transformation primary equipment maintenance scheme still has some room for optimization. In addition, it's necessary to note that maintenance yield is not only limited to the same year, but should be a long process. This issue does not calculate the future earnings, so this significantly causes low investment revenue and need further improvement in the future.

\section{Summary}

On the basis of system risk, from the economic, safety and environment and other aspects quantitatively evaluate power transmission and transformation equipment maintenance schemes and obtain comprehensive Evaluation results, which provide a reasonable basis for guiding the allocation of maintenance resources and optimizing the maintenance schemes.

\section{References}

[1] Howard W. Penrose, Simple time-to-failure estimation techniques for reliability and maintenance of equipment, IEEE Electrical Insulation Magazine. Vol. 20, No. 7, 2009, pp.14-18.

[2] Xiong Hao,Sun Caixin,Zhang Yun,Tan Zhihong,Dai Yao,A Hierarchical Grey Evaluation Model for Operation Condition of Power Transformers,Automation of Electric Power Systems, vol. 31,2007,pp.55-60..

[3] A. Grall, C. Berenguer, L. Dieulle, A condition-based maintenance policy for stochastically deteriorating systems, Reliability Engineering and System Safety,2002,pp.167-180.

[4] Liao Ruijin,Wang Qian,Luo Sijia1,Liao Yuxiang,Sun Caixin,Condition assessment model for power transformer in service based on fuzzy synthetic evaluation,Automation of Electric Power Systems, vol. 32,2008,pp.70-75.

[5] Zhang Yaohui Guo Jinmao Xu Zongchang,Failure Risk Based Determination of the Test Intervals for Condition Based Maintenance, Chinese Society for Electrical Engineering, vol. 19, 2008, pp.555-557.

[6] An L ing,Jiang Xiuchen,Zhu Yu,Han Zhendong,Chen Yazhu, Object-oriented design and implementation of the state maintenance software system for transmission lines,Automation of Electric Power Systems, Apr.10, 2002,pp. 66-69. 
[7] Yuan Zhijian,Sun Caixin,Li Jian,Du Lin,Liao Ruijin, Study on condition-based maintenance policy of transformer based on fuzzy multiple expert and multiple attribute group decision making,Automation of Electric Power Systems, 2004,pp.66-70.

[8] Zhang huai-yu,Zhu song-lin,Zhang yang,Lou qi-min,Zhang liang,Research and Implementation of Condition-Based Maintenance Technology System for Power Transmission and Distribution Equipment, Power System Technology, vol. 33, 2009,pp.70-73. 